\title{
Electronic structure of $\mathrm{BiFeO}_{3}$ in the presence of strong electronic correlations
}

\author{
L. Craco, ${ }^{1,2}$ S. S. Carara, ${ }^{1}$ and S. Leoni ${ }^{3}$ \\ ${ }^{1}$ Instituto de Física, Universidade Federal de Mato Grosso, 78060-900, Cuiabá, MT, Brazil \\ ${ }^{2}$ IFW Dresden, Institute for Solid State Research, P.O. Box 270116, D-01171 Dresden, Germany \\ ${ }^{3}$ School of Chemistry, Cardiff University, Cardiff, CF10 3AT, United Kingdom
}

(Received 26 June 2018; revised manuscript received 1 November 2018; published 7 January 2019)

\begin{abstract}
Using density-functional dynamical mean-field theory (DFDMFT) we show the importance of multiorbital electronic correlations in determining the insulating state of $\mathrm{BiFeO}_{3}$, a multiferroic material with an electron band gap larger than its bare bandwidth. Within the $\mathrm{Fe}^{3+}$ oxidation state and using realistic values for the on-site Coulomb interaction, we unveil strongly correlated key features probed in x-ray photoelectron and absorption spectra, showing good qualitative theory-experiment agreement. We explore the electronic reconstruction hidden in ferromagnetic $\mathrm{BiFeO}_{3}$, predicting broad orbital- and spin-polarized features at low temperatures. Our proposal for ferromagnetic $\mathrm{BiFeO}_{3}$ is expected to be an important step to understanding the emergent correlated electronic structure of magnetoelectronic and spintronic materials with persisting ordered localized moments coexisting with Coulomb reconstructed electronic states.
\end{abstract}

DOI: 10.1103/PhysRevB.99.045112

\section{INTRODUCTION}

The coupling between orbital, lattice, and spin degrees of freedom provides challenges for designing novel advanced functional materials, both on fundamental and applied perspectives. In this context, perovskite multiferroics, in which electric and magnetic orders coexist in the same structural phase, have attracted great interest in recent years [1]. Particularly interesting are bismuth-based perovskites [2-5]. Due to the large orbital radius of the $\mathrm{Bi}-6 s^{2}$ lone pairs, the crystal structure of Bi perovskites is usually distorted to low symmetries, producing spontaneous ferroelectric polarization along certain crystallographic directions [6]. Among the $\mathrm{Bi}$ perovskites, $\mathrm{BiFeO}_{3}$ is the most systematically studied system both from theory [7-14] and experimental $[3,4,15-24]$ perspectives.

The lattice of $\mathrm{BiFeO}_{3}$ crystal (Fig. 1) is rhombohedrally distorted with unit cell parameters $a=b=c=5.634 \AA$ and $\alpha=59.348^{\circ}$ [17]. By moving $\mathrm{Bi}$ and $\mathrm{Fe}$ atoms along the pseudocubic [111] direction and $\mathrm{O}$ atoms along the pseudocubic $\langle 110\rangle$ direction, the unit cell elongates with the neighboring octahedrons rotated in different directions along the [111] crystallographic axis [4]. At ambient pressure conditions, $\mathrm{BiFeO}_{3}$ has a ferroelectric Curie temperature $T_{C}$ of $1103 \mathrm{~K}$, [16], and an antiferromagnetic Néel temperature $T_{N}$ of $643 \mathrm{~K}$ [15]. The Fe spins form a $G$-type antiferromagnetic ordered structure, in which the spins are coupled ferromagnetically in the pseudocubic (111) planes and antiferromagnetically between neighboring (111) planes, so that each Fe atom couples antiferromagnetically with its nearest $\mathrm{Fe}$ neighbors [25]. Bulk $\mathrm{BiFeO}_{3}$ also reveals weak ferromagnetic order (of the Dzyaloshinskii-Moriya type) [7] at low temperatures, which mainly results from the canting of the antiferromagnetic sublattice with spiral spin structure [18]. Interestingly, this spin circular cycloid state can be suppressed at room temperature [20], where the coexistence of ferroelectricity and ferromagnetism makes $\mathrm{BiFeO}_{3}$ a potential multiferroic material for future applications on magnetoelectric and memory devices [26].

Before delving into the details of the strongly correlated electronic structure of paramagnetic and ferromagnetic $\mathrm{BiFeO}_{3}$, we remark that bismuth ferrite is expected to be a potential functional material where ferroelectricity and ferromagnetism coexist at room temperature [2]. Although ferroelectricity and antiferromagnetism have long been known in $\mathrm{BiFeO}_{3}$ single crystals [27], different experimental approaches have been conducted in recent years to explore the formation of ferromagnetic $\mathrm{BiFeO}_{3}$ bulk crystals, films $[2,20,28]$, and heterostructures [29]. Importantly, the latter study is providing direct evidences that the formation of interface ferromagnetism is related to electronic orbital reconstruction. Theoretically, magnetically induced electronic reconstruction in $\mathrm{BiFeO}_{3}$ has been investigated using firstprinciples density functional theories (DFT) $[4,8,10,23]$. Particularly relevant in this context is the recent density functional dymanical mean-field theory (DFDMFT) study by S. Paul et al. [14] (see our discussion below), where the spectral properties of paramagnetic and antiferromagnetic phases of $\mathrm{BiFeO}_{3}$ were investigated and compared to photoemission spectra. However, to the best of our knowledge, there have been no previous DFDMFT investigations on the interplay between dynamical multiorbital (MO) interactions, oxidation state, and ferromagnetism, all inducing electronic orbital reconstruction in $\mathrm{BiFeO}_{3}$. A key aspect of the present study is thus to shed new light onto this problem of fundamental and applied interest, showing intrinsic MO fingerprints relevant for future experiments on bulk and $\mathrm{BiFeO}_{3}$ films. Strong, local orbital interactions are shown to be the underlying reason for the correlation features seen in optical and spectroscopy experiments. As a byproduct of our analysis, we predict broad orbital- and spin-polarized features, which could be seen in 


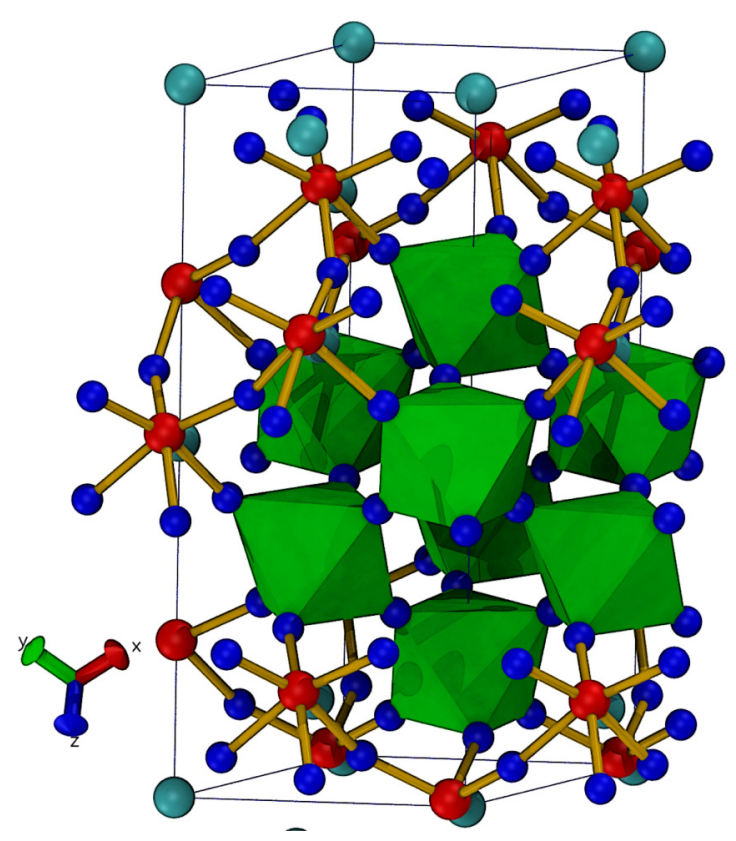

FIG. 1. Crystal structure of $\mathrm{BiFeO}_{3}$. Large turquoise, medium red, and small blue spheres represent $\mathrm{Bi}, \mathrm{Fe}$, and $\mathrm{O}$ atoms, respectively.

future experiments on bulk and ferromagnetic thick films. Within our DFDMFT treatment, we identify one relevant among various types of mechanisms, which would lead to changes in the one-particle spectra of ferromagnetic $\mathrm{BiFeO}_{3}$, that is, the effect of spin ordering in determining the MO electronic state relevant for magnetoeletronics [30]. In this contribution, we show how the majority and minority spin parts of the correlated spectra are shifted by different amounts depending on the orbital and spin character due to strong MO many-body scattering processes. To our knowledge, this is the first study showing such nontrivial orbital- and spin-selective dynamical transfer of spectral weight across the magnetic phase transition of $\mathrm{BiFeO}_{3}$.

Bulk $\mathrm{BiFeO}_{3}$ is known to be a wide band gap Mott insulator above $T_{N}[13,14]$. The gap value obtained from correlated [generalized gradient approximation plus dynamical meanfield theory (GGA+DMFT)] band structure calculations for the rhombohedral $R 3 c$ structural phase was found to be close to $1.2 \mathrm{eV}$ [13]. Albeit smaller compared with the experimental value of $1.8 \mathrm{eV}$ for the indirect optical gap at ambient pressures [31], this result is in very good agreement with the indirect band gap value of $1.3 \mathrm{eV}$ measured in thin films [32]. According to this GGA+DMFT study, the magnetic moment of $\mathrm{BiFeO}_{3}$ is $4.6 \mu_{B}$ at ambient pressure conditions, consistent with a high-spin $(S=5 / 2)$ state.

The electronic structure, band gap, and magnetic properties of bulk and $\mathrm{BiFeO}_{3}$ films were investigated by different $a b$ initio density functional theories implemented in the GGA or local density approximation (LDA) as well as $($ GGA/LDA $)+U$ and local spin density approximation plus $U(\mathrm{LSDA}+U)$ schemes [4,8-10,12,22,23]. A perusal of these $\mathrm{DFT}+U$ studies, which explicitly take into account the effect of local electron-electron interactions, reveal that the on-site Coulomb interaction $U$ (effective or not) for $\mathrm{BiFeO}_{3}$ could be in the range between 2 and $9 \mathrm{eV}$ [8,10,22]. However, the most common values for $\mathrm{BiFeO}_{3}$ crystals and films are in the range between 5 and $7 \mathrm{eV}$, which is consistent with $U=$ $6.0 \mathrm{eV}$ used in the GGA+DMFT study of insulator-metal transition of compressed $\mathrm{BiFeO}_{3}$ [13]. However, in spite of these theoretical efforts, neither the changes of the Mott insulating state of paramagnetic $\mathrm{BiFeO}_{3}$ with increasing $U$ nor the electronic spectrum [22,24] hidden in the ferromagnetic ordered state of bulk [20] and thick $\mathrm{BiFeO}_{3}$ films [11] were addressed so far. In this work, we shed light onto this problem within LDA+DMFT approximation [33], showing that the emergent strongly correlated electronic state is caused by large transfer of spectral weight with concomitant formation of orbital-selective localized moments in $\mathrm{BiFeO}_{3}$.

Recently, Paul et al. [14] have used the LDA+DMFT approach to compute the valence-band photoelectron and magnetic excitation spectra of $\mathrm{BiFeO}_{3}$. The results of this study were shown to be in good agreement with the experimental data [22] for the occupied states, whereas the LDA $+U$ method fails to capture the general features of the measured spectra. This study highlights the importance of incorporating dynamical many-particle aspects of MO electronic correlation effects within the $\mathrm{Fe}-3 d$ orbitals to correctly reproduce the experimental excitation spectra. Likewise, here we present a self-consistent study of electronic structure calculations using the $\mathrm{LDA}+\mathrm{DMFT}$ approach for $\mathrm{BiFeO}_{3}$ parent compound. The calculated density-of-states (DOS) is compared with experimental photoemission (PES) [14,22] and inverse photoemission spectroscopy (IPES) [24] data. We show how this can provide a consistent explanation for the more relevant electron correlation fingerprints in the occupied and unoccupied states as well as to optical-absorption spectra of $\mathrm{BiFeO}_{3}$ singlecrystal [21] within the same theoretical framework.

\section{THEORY, RESULTS, AND DISCUSSION}

Within the rhombohedral (space group $R 3 c$ ) crystal structure and using lattice constants and atomic positions at ambient pressure conditions [17], one-electron band structure calculations based on local density approximation (LDA) and local spin density approximation (LSDA) were performed for paramagnetic and ferromagnetic $\mathrm{BiFeO}_{3}$ using the linear muffin-tin orbitals (LMTO) scheme, in the atomic sphere approximation [34]. The LMTO scheme [35] provides reliable results at one-particle level and has been used to study the electronic structure of different materials [36], including perovskite titanate oxides [37,38], and iron-based systems [39]. Here, self-consistent solutions for para- and ferromagnetic $\mathrm{BiFeO}_{3}$ were reached by performing calculations with 560 irreducible $\mathbf{k}$ points. The radii of the atomic spheres were chosen as $r=3.16(\mathrm{Bi}), r=2.46(\mathrm{Fe})$, and $r=1.96(\mathrm{O})$ a.u. in order to minimize their overlap.

Consistent with previous DFT calculations for the nonmagnetically ordered phase of $\mathrm{BiFeO}_{3}$ [40], our LDA result in Fig. 2 shows that the active electronic states in this Biperovskite compound involve the Fe $3 d$ carriers, where all $d$ bands have appreciable weight near the Fermi energy, $E_{F}=$ $\omega=0$. As seen, in the distorted $R 3 c$ phase, the five Fe orbitals split into a nondegenerated $t_{2 g^{1}}$ band and two twofold degenerated $t_{2 g^{2}}$ and $e_{g^{2}}$ bands [41]. As expected for perovskite 


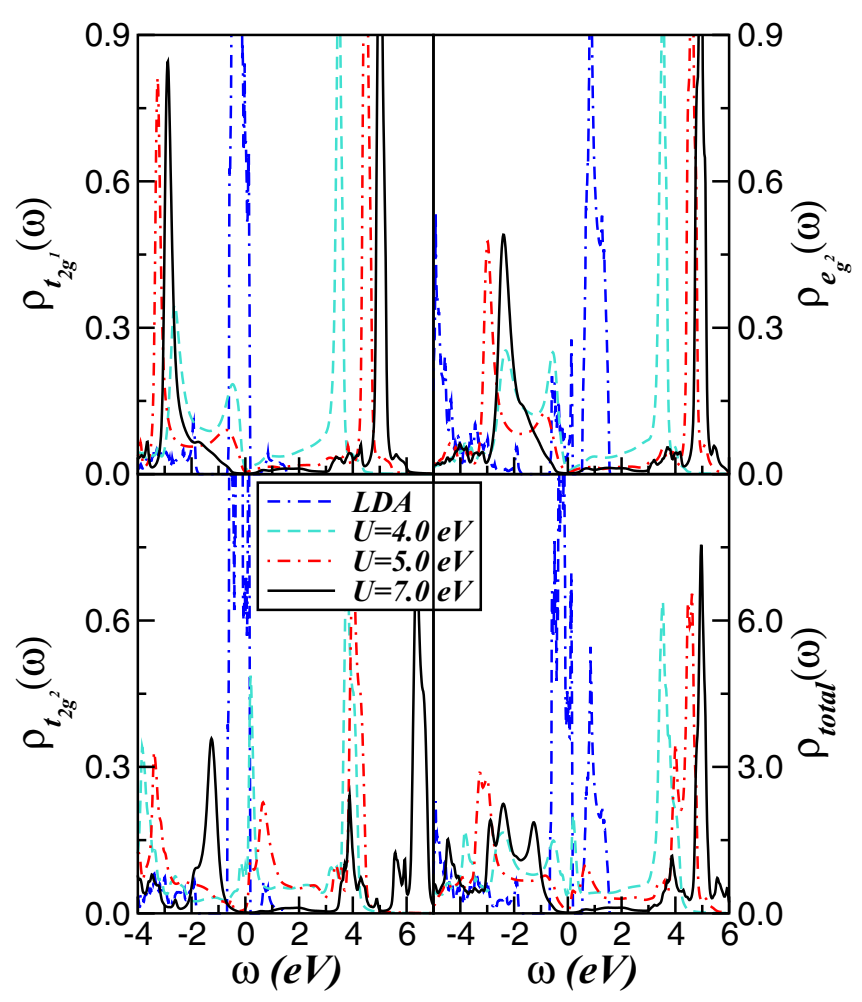

FIG. 2. Orbital-resolved LDA and LDA+DMFT density-ofstates (DOS) for the $\mathrm{Fe} 3 d$ orbitals of paramagnetic $\mathrm{BiFeO}_{3}$. An important feature to be seen is the narrow bare bandwidth and the fact that all bands in LDA span a small region near the Fermi level, $E_{F}=\omega=0$. This confirms that the electronic states relevant to Bismuth ferrite [13] are $\mathrm{Fe} 3 d$ states. Noteworthy is the electronic reconstruction and the formation of sharp Hubbard satellites with increasing on-site Coulomb interaction $U$.

transition-metal oxides, the $e_{g^{2}}$ states are higher in energy and almost fully polarized within LDA. However, as shown below and similar to previous LDA+DMFT calculations for low-pressure $\mathrm{BiFeO}_{3}$ [13], strong $\mathrm{MO}$ electron-electron interactions will scatter electrons among different orbital channels via the interorbital Coulomb interaction $U^{\prime}$ and the Hund's exchange parameter $J_{H}$, lowering the electronic $e_{g^{2}}$ states towards the valence band and thus reducing the large LDA orbital polarization. Importantly, the narrow bare bandwidth $W$ (which is below $1 \mathrm{eV}$ for all active Fe orbitals) in LDA puts $\mathrm{BiFeO}_{3}$ among the strongest Mott-localized transition-metal perovskites [37,42].

Although first-principles (GGA/LDA/LSDA)+U calculations have provided reliable structural and magnetic groundstate information for $\mathrm{BiFeO}_{3}[4,8-12]$, they are known to generically fail to capture the ubiquitous dynamical correlation effects [14], and so cannot access MO Mott localization and the emergence of Hubbard satellites (local moments) at high energies. Combining DFT with DMFT is the state-ofthe-art prescription for overcoming this problem [33]. Thus, as common to correlated materials within GGA/LDA, the oneelectron part of the $\mathrm{MO}$ Hamiltonian for $\mathrm{BiFeO}_{3}$ reads $H_{0}=$ $\sum_{\mathbf{k}, a, \sigma} \epsilon_{a}(\mathbf{k}) c_{\mathbf{k}, a, \sigma}^{\dagger} c_{\mathbf{k}, a, \sigma}$, where $a=\left(t_{2 g^{1}}, t_{2 g^{2}}, e_{g^{2}}\right)$ denote its diagonalized $3 d$ orbitals and $\epsilon_{a}(\mathbf{k})$ is the corresponding band dispersion, which encodes details of the one-electron band structure. These five Fe- $3 d$ bands are the relevant one-particle inputs for MO-DMFT, which generates a Mott-Hubbard insulating state with a large band gap at $U=7.0 \mathrm{eV}$ as shown below. Similar to Ref. [13], the correlated many-body Hamiltonian for $\mathrm{BiFeO}_{3}$ reads $H_{\text {int }}=U \sum_{i, a} n_{i, a, \uparrow} n_{i, a, \downarrow}+$ $U^{\prime} \sum_{i, a \neq b} n_{i, a} n_{i, b}-J_{H} \sum_{i, a \neq b} \mathbf{S}_{i, a} \cdot \mathbf{S}_{i, b}$. (Here, $U$ is the onsite Coulomb interaction, $U^{\prime}=U-2 J_{H}$ is the interorbital Coulomb interaction term, and $J_{H}$ is the Hund's coupling.) We evaluate the many-particle Green's functions $\left[G_{a, \sigma}(\mathbf{k}, \omega)\right]$ of the MO Hamiltonian $H=H_{0}+H_{\text {int }}$ within LDA+DMFT [33], using MO iterated perturbation theory (MO-IPT) as impurity solver [43]. The DMFT solution involves replacing the lattice model by a self-consistently embedded MO-Anderson impurity model, and the self-consistency condition requiring the local impurity Green's function to be equal to the local Green's function for the lattice. The full set of equations for the MO case can be found in Ref. [43] so we do not repeat the equations here.

It is worth mentioning here that the IPT is an interpolative ansatz that connects the two exactly soluble limits of the oneband Hubbard model [44], namely, the uncorrelated $(U=0)$ and the atomic $[\epsilon(\mathbf{k})=0]$ limits. It accounts for the correct low- and high-energy behavior of the one-particle spectra, and the metallic Fermi liquid behavior in the large- $D$ limit (DMFT) [45]. It ensures the Mott-Hubbard metal-insulator transition from a correlated Fermi liquid metal to a MottHubbard insulator as a function of the Coulomb interaction $U$. The IPT is known to be computationally very efficient, with real frequency output at zero and finite temperatures. As shown below, the LDA+DMFT(MO-IPT) solution for $\mathrm{BiFeO}_{3}$ introduces nontrivial effects stemming from the dynamical nature of strong electronic correlations. These processes namely lead to large transfer of spectral weight across large energy scales in response to changes in the on-site Coulomb repulsion, a characteristic lying at the heart of the anomalous responses of correlated electron systems. We recall that similar perturbative scheme as used here for the MO Hamiltonian of $\mathrm{BiFeO}_{3}$ has been proposed by P. Pou et al. [46], where electron correlation effects in local-orbital electronic structure calculations were applied to Si-bulk crystals and $\mathrm{H}_{2} \mathrm{O}$ molecules. It is also worth noting that direct comparisons between MO-IPT results with numerically exact methods, like the continuous-time quantum Monte Carlo (CTQMC) have been performed in recent years. In their detailed work, Dasari et al. [47] provided an extensive comparison of MO-IPT results and data from CT-QMC method, showing good agreement between the two impurity solvers. Hence, in view of this and our previous studies on Fe-based systems showing good theory-experiment agreement [39], we are confident to use MO-IPT to explore the correlated electronic structure of para- and ferromagnetic $\mathrm{BiFeO}_{3}$.

Let us now discuss our L(S)DA+DMFT(MO-IPT) results obtained within the formal $d^{5}$ electronic configuration of the $\mathrm{Fe}^{3+}$ oxidation state in $\mathrm{BiFeO}_{3}$. In Fig. 2, we display the effect of increasing local electron-electron interactions on the orbital-resolved spectral functions of $R 3 c \mathrm{BiFeO}_{3}$ parent compound. As seen at $U=4.0 \mathrm{eV}$ (and $J_{H}=0.7 \mathrm{eV}$ ), the system is an orbital-selective metal [48], where only the $t_{2 g^{2}}$ orbital sector display appreciable spectral weight at $E_{F}$. In spite of this selective metallic behavior, lower (LHB) and 
upper (UHB) Hubbard bands at high energies are visible in a more or less pronounced way, depending on the orbital character, in all orbital-resolved spectral functions. This behavior highlights the intrinsic tendency towards large transfer of spectral weight and Mott localization in transition-metal oxide compounds [42], where $U / W$ ratio is usually sizable. Moreover, at $U=5.0 \mathrm{eV} \mathrm{BiFeO}_{3}$ is in the all-orbital Mott localized state with an orbital-selective band gap size followed by incoherent shoulder features above and below $E_{F}$. As seen in Fig. 2, the LDA+DMFT spectral functions are highly reshaped by electron-electron interactions; at $U=7.0 \mathrm{eV}$ most of the spectral weight of $\mathrm{BiFeO}_{3}$ is located at narrow Hubbard satellites, and only depleted shoulder states relevant optical conductivity responses are found at energies above the Mott gap.

To provide further insights for the emergent electronic reconstruction and strong Mott localization in rhombohedral $\mathrm{BiFeO}_{3}$, we have computed its orbital-resolved optical conductivity response using the correlated spectral functions for $U=7.0 \mathrm{eV}$, shown in Fig. 2. In the large dimensional limit (DMFT), this quantity is directly evaluated as convolution of the one-particle Green's functions [33], and for $\mathrm{MO}$ correlated electron systems like $\mathrm{BiFeO}_{3}$, the real part of the optical conductivity tensor can be evaluated as $\sigma_{a, \sigma}(\omega)=v_{a}^{2} \frac{2 \pi e^{2} \hbar}{V} \sum_{\mathbf{k}} \int d \omega^{\prime} \frac{f\left(\omega^{\prime}\right)-f\left(\omega+\omega^{\prime}\right)}{\omega} A_{\mathbf{k}, a, \sigma}\left(\omega^{\prime}+\right.$ $\omega) A_{\mathbf{k}, a, \sigma}\left(\omega^{\prime}\right)$ [49], where $V$ is the volume of the unit cell per formula unit, $v_{a}$ is the fermion velocity in orbital $a$ and $A_{a}(\mathbf{k}, \omega)$ is the corresponding fully renormalized one-particle spectral function.

In Fig. 3, we show the orbital-resolved optical conductivity $\left[\sigma_{a}(\omega)\right]$ of $\mathrm{BiFeO}_{3}$. As seen, the smallest optical gap, of $\simeq 0.9 \mathrm{eV}$, is found within the $t_{2 g^{2}}$ orbital sector, which in-

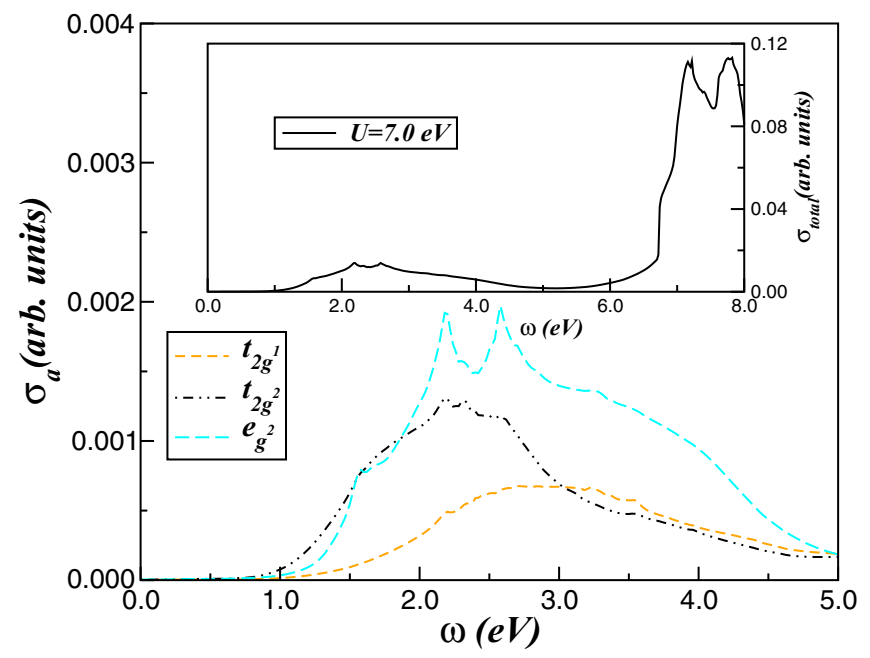

FIG. 3. LDA+DMFT $\left(U=7.0 \mathrm{eV}, J_{H}=0.7 \mathrm{eV}\right)$ orbital-resolved optical conductivity of $\mathrm{BiFeO}_{3}$. Notice the low-energy line shape and optical gaps, which are in qualitative good accord with polarized optical-absorption spectra of $\mathrm{BiFeO}_{3}$ single-crystal [21]. The inset displays our results in a large frequency window, showing an intense enhancement of the optical conductivity response at energies above $6.7 \mathrm{eV}$ originated from optical excitations between the Hubbard satellites and the low energy shoulders in the LDA+DMFT spectral functions. creases up to $1.2 \mathrm{eV}$ for the more Mott localized $t_{2 g^{1}}$ orbital at $U=7.0 \mathrm{eV}$. While there is no strict one-to-one correspondence between optical conductivity and optical-absorption spectra, our results are in qualitative good accord with extant polarized data for the absorption edged below $1.4 \mathrm{eV}$ [21]. Similar optical low-energy line shape and redistribution of optical spectral weight among different $3 d$ orbitals shown in Fig. 3 were reported few years ago for the titanate perovskite $\mathrm{YTiO}_{3}$ both on theory [38] and experiments [50]. In particular, the peak structure close to $2.0 \mathrm{eV}$ seen in this strongly correlated $d^{1}$ transition-metal oxide has been identified as an effect arising from interorbital Mott-Hubbard excitations, and this scenario holds true also for the $d^{5}$ electronic state of $\mathrm{BiFeO}_{3}$. Moreover, also in close analogy to $\mathrm{YTiO}_{3}$ [50], large-scale $[\geqslant O(7.0) \mathrm{eV}]$ transfer of optical spectral weight (see inset of Fig. 3) between the low-energy shoulders above the Mott gap and the narrow Hubbard satellites at high energies is obtained for total optical conductivity $\left[\sigma_{\text {total }}(\omega)=\sum_{a, \sigma} \sigma_{a, \sigma}(\omega)\right]$ of $\mathrm{BiFeO}_{3}$ and future optical conductivity studies are called for to corroborate our prediction.

Room-temperature Mössbauer spectrum of $\mathrm{BiFeO}_{3}$ nanoparticles reveal two quadrupole doublets, which were assigned to coexisting $\mathrm{Fe}^{3+}$ and $\mathrm{Fe}^{2+}$ valence states [23]. The degree of amorphization (or metamictization) in $\mathrm{BiFeO}_{3}$ nanoparticles was found to be $\mathrm{Fe}_{0.838}^{3+} \mathrm{Fe}_{0.162}^{2+}$ [23], a ratio consistent with $\mathrm{x}$-ray photoelectron spectroscopy (XPS) were $19 \% \mathrm{Fe}$-ions are in the $d^{6}\left(\mathrm{Fe}^{2+}\right)$ electronic configuration [19]. The changes in the $\mathrm{Fe}$-ionic content in $\mathrm{BiFeO}_{3}$ might be due to the multiple valences possible for $\mathrm{Bi}$ and $\mathrm{Fe}$, particularly if the $\mathrm{Bi}$ ions acquire an average formal valence of $4+$, leaving the Fe ion with a $2+$ electronic state [8]. With this in mind as well as the fact that heating $\mathrm{BiFeO}_{3}$ seems to enhance $\mathrm{Fe}^{3+} / \mathrm{Fe}^{2+}$ valence fluctuations of the $\mathrm{Fe}$ ion [51], in the main panel of Fig. 4, we compare the LDA+DMFT total spectral functions obtained for $\mathrm{Fe}^{3+}$ and $\mathrm{Fe}^{2+}$ valence states. Electron-electron interactions are expected to reach their maximum at half-filling (or within the $d^{5}$ electronic configuration of the Fe $3 d$ shell) and to decrease away from this configuration [52]. In spite of the large $t_{2 g}-e_{g}$ band splitting in LDA, this electronic behavior is consistent with our results in Fig. 4 where strong correlation fingerprints are found within the $\mathrm{Fe}^{3+}$ valence state. Interestingly, while the energy difference between the Hubbard bands is reduced, the size of the Mott-Hubbard gap remains nearly unaffected with increasing total electron concentration of the $\mathrm{Fe} 3 d$ shell. Moreover, in the inset of Fig. 4, we display the total one-particle spectral function of $\mathrm{BiFeO}_{3}$ solid solution with $19 \%$ of metamictization [19], showing that additional low energy features would be seen in future spectroscopy and tunneling experiments.

Since the oxidation state of $\mathrm{BiFeO}_{3}$ systems remains uncertain $[19,23,53]$, for a detailed analysis of dynamical MO electronic interactions we compare our LDA+DMFT results in Fig. 4 with experimental hard x-ray photoelectron spectroscopy (HAXPES) of bulk [14] and $\mathrm{BiFeO}_{3}$ thick films [22] as well as with $\mathrm{O} K$-edge $\mathrm{X}$-ray absorption spectra (XAS) [24]. In view of the fact that only the five $3 d$ bands have been included in the LDA+DMFT treatment, we focus on the energy window (which, however, is rather wide) $-3.0 \leqslant$ $\omega \leqslant 5.0 \mathrm{eV}$ around $E_{F}$ : this is beyond the region where only 


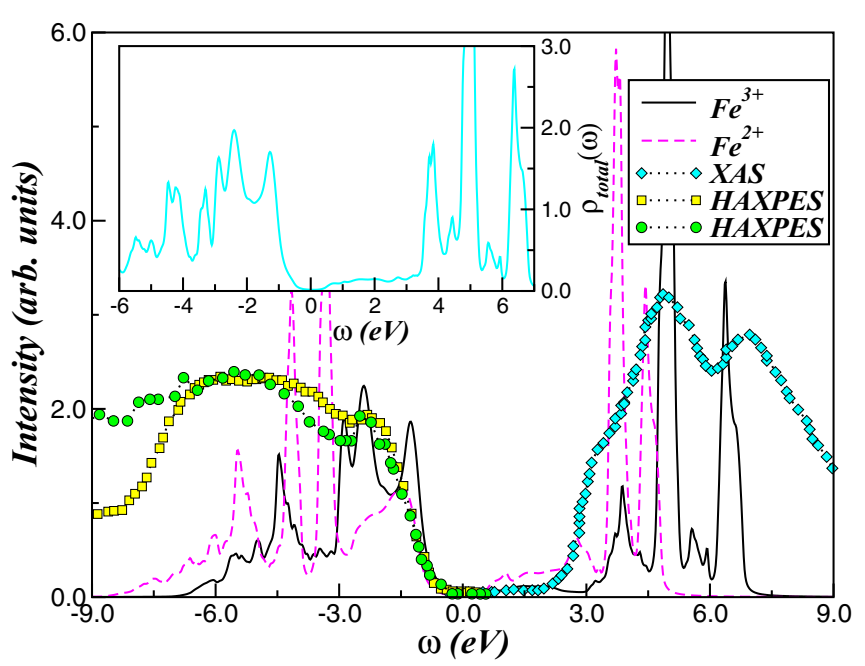

FIG. 4. Comparison between the total $3 d$ LDA+DMFT DOS for the $\mathrm{Fe}^{3+}$ and $\mathrm{Fe}^{2+}$ oxidation states with experimental hard x-ray photoelectron spectroscopy (HAXPES) of bulk (square) [14] and $\mathrm{BiFeO}_{3}$ thick films (circle) [22] as well as with $\mathrm{O} K$-edge $\mathrm{x}$-ray absorption (XAS) spectra (diamond) [24], showing good qualitative theoryexperiment agreement at energies between $-3.0 \leqslant \omega \leqslant 5.0 \mathrm{eV}$ is visible. In particular, the low-energy line shape in HAXPES and the double-bump feature in XAS are accurately resolved in the LDA+DMFT spectrum for $U=7.0 \mathrm{eV}$. Inset shows the total LDA+DMFT spectral function to be seen in future experiments of $\mathrm{BiFeO}_{3}$ with $19 \%$ of metamictization [19].

the five $d$ bands dominate in LDA [14,54]. As seen, good quantitative agreement with HAXPES data is obtained at low energies. Here, the bump feature close to $2.5 \mathrm{eV}$ binding energy in HAXPES [14,22] is interpreted as arising from collective intra- and interorbital fluctuations within the strongly correlated Mott insulating state. However, similar to other correlated transition-metal compounds [55], due to strong dynamical correlation effects in $\mathrm{BiFeO}_{3}$, the $t_{2 g^{1}}$ and $e_{g^{2}} \mathrm{LHBs}$ for $U=7.0 \mathrm{eV}$ are shifted toward energies where the $\mathrm{O}-2 p$ and Bi- $6 p$ bands start to occur in the LDA [14], i.e., below $1.8 \mathrm{eV}$ binding energy (see Fig. 2). This superposition of states with $p$ and $d$ orbital character together with background signals [14] present in the experimental data makes it difficult to compare our LDA+DMFT results with the valence band spectra of $R 3 c \mathrm{BiFeO}_{3}$. Thus more work is needed to identify the orbital character relevant to band-gap suppression at low binding energies in doped $\mathrm{BiFeO}_{3}$. Remarkable as well is the fact that our LDA+DMFT result for the $\mathrm{Fe}^{3+}$ valence state is also in qualitative good agreement with XAS data for $\mathrm{BiFeO}_{3}$ [24]. In particular, the two pronounced $t_{2 g}-e_{g}$ peaks and their energy separation are faithfully reproduced. Only the line shape of the pre-edged peak close to $3.0 \mathrm{eV}$ above $E_{F}$ is not well captured as compared to experiment. However, according to our results in Fig. 4, it is plausible to assume that the preedged peak reflects intrinsic tendency towards metamictization with small amount of $\mathrm{Fe}^{2+}$ valence and conduction band states in the total electronic structure probed in spectroscopy experiments. Taken together, our theory-experiment comparison constitute a consistent, quantitative rationalization of basic strongly correlated one-particle fingerprints and serve as a basis to explore the orbital reconstruction in ferromagnetic $\mathrm{BiFeO}_{3}[11,20,23]$, as shown below.

It is instructive to note, however, that, though quantitatively good, the agreement is not quite as perfect in the high-energy valence and conduction band regions. Particularly, consideration of the spectrum for energies below the $3.0 \mathrm{eV}$ binding energy in HAXPES and for $\omega>7.0 \mathrm{eV}$ in $\mathrm{XAS}$ is limited by our restriction to the $3 d$ sector. Since in our LDA+DMFT calculations we have ignored contributions from the O-2 $p$ bands $[12,14]$, we cannot account for spectral weight changes at high binding energies. We also see that some spectral weight is lost in the XAS spectra, possibly due to the lack of $\mathrm{Bi}^{3+}$ bands [12]. Thus our work should be regarded as an attempt toward a more concrete LDA+DMFT description of correlation-induced strong electronic reconstruction in $\mathrm{BiFeO}_{3}$ and related Mott insulating compounds. An additional possible physical reason for the theory-experiment discrepancy is the lack of core-hole scattering [56], which may induce additional broadening effects in our theoretical results [57]. Core-hole effects will introduce additional incoherent excitations (which are usually probed by $x$-ray absorption experiments), substantially modifying the many-body character of the unoccupied electronic states at moderate to high energies. Thus, to shed more light on the dynamical many-particle responses of paramagnetic $\mathrm{BiFeO}_{3}$, future DFT+DMFT studies should incorporate the oxygen $p$ bands as well as the local core-hole potential [56] in the multiband problem of $\mathrm{BiFeO}_{3}$.

In earlier studies, it has been shown how the correlated electronic state changes across different magnetic [38,58] phase instabilities in MO systems, and why they can be semiquantitatively understood using (GGA/LDA)+DMFT with sizable MO correlations. Here, we extend this aspect to characterize the electrical properties of ferromagnetic $\mathrm{BiFeO}_{3}$ and its link to spin- and orbital-selective metallicity. The latter is relevant for ferromagnetic bulk and thick films, where reduced dimensionality and surface effects are expected not to play a detrimental role for the already strongly correlated electronic structure. To begin with, in the two main panels of Fig. 5, we show the LSDA DOS of the majority ( $\uparrow)$ and minority $(\downarrow)$ spin channels for ferromagnetic $\mathrm{BiFeO}_{3}$. As expected for ferromagnetic systems, the majority spin band is transferred to lower binding energies across the magnetic phase transition, while the minority spin channel gets depopulated due to large spin splitting. Interesting, however, is the fact that the band broadening is almost not seen in the minority $t_{2 g}$ channel, which remains close to that found in the paramagnetic state, albeit transferred to energies above $E_{F}$. On the other hand, an effective $100 \%$ broadening is obtained for the minority $e_{g}$ sector. Taken together, our LSDA results in Fig. 5 suggest strong spin-orbital differentiation across the para- to ferromagnetic phase transition in $\mathrm{BiFeO}_{3}$ which would lead to decreased electron correlation through increasing bandwidth, an undoubtedly significant feature to be seen in future experiments. The central result to be seen in Fig. 5 is the band broadening modification observed in the LSDA DOS, which as a consequence is expected to induce partial screening of the Coulomb potential [59] across the paramagnetic-to-ferromagnetic quantum phase transition in $\mathrm{BiFeO}_{3}$. Motivated thereby, we have chosen $U=6.0 \mathrm{eV}$ [9] as representative value Coulomb 


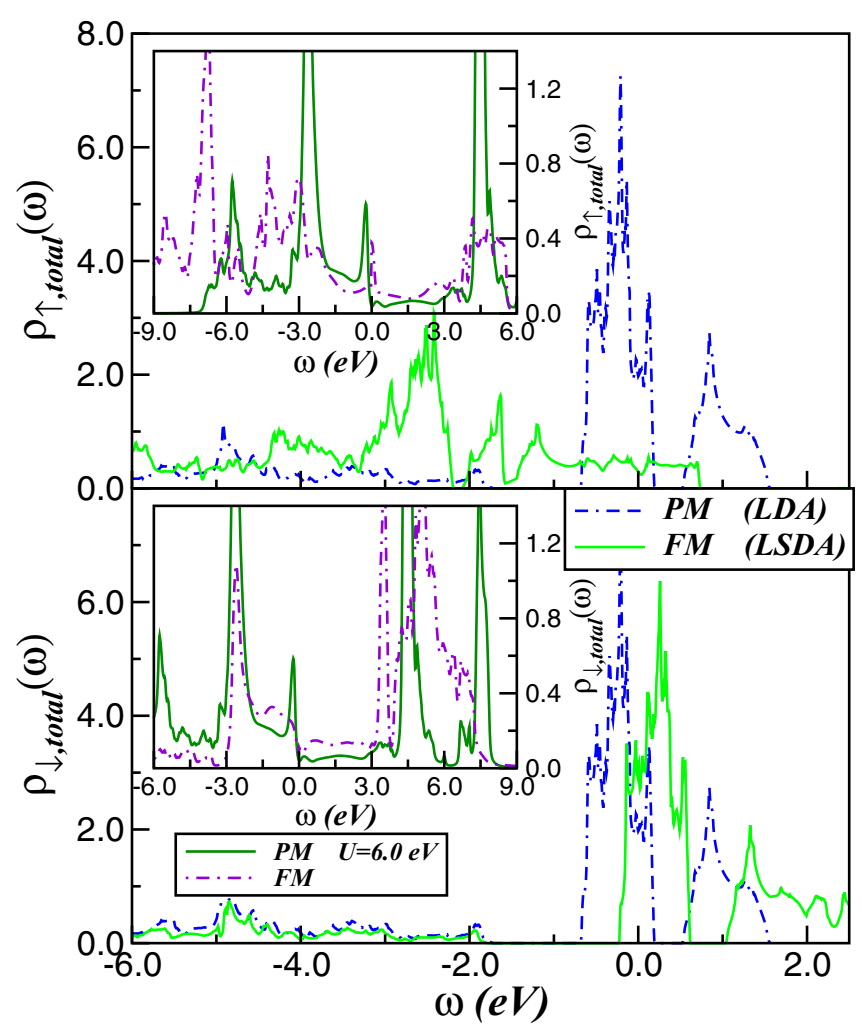

FIG. 5. Spin-resolved L(S)DA total spectral functions of paramagnetic (dotted lines) and ferromagnetic (solid lines) $\mathrm{BiFeO}_{3}$ parent compound. Notice the ferromagnetism-induced broad bandwidth in majority spin- $\uparrow$ channel and robustness of the minority $t_{2 g}$ line shape against ferromagnetic spin ordering. Inset shows the changes in the $\mathrm{L}(\mathrm{S}) \mathrm{DA}+\mathrm{DMFT}$ spin-resolved spectral functions across the para- to ferromagnetic transition of $\mathrm{BiFeO}_{3}$.

interaction parameter for ferromagnetic $\mathrm{BiFeO}_{3}$. The insets of Fig. 5 show how LSDA+DMFT modifies the LSDA band structure. As expected, MO dynamical correlations arising from $U, U^{\prime}$, and $J_{H}$ lead to spectral weight redistribution over large energy scales within the correlated MO electronic states of ferromagnetic $\mathrm{BiFeO}_{3}$.

According to our results in Fig. 6, metallicity in ferromagnetic $\mathrm{BiFeO}_{3}$ is of orbital-selective type, where electronic localization is restricted to a particular subset of the active orbitals present in the problem. This state usually has Mott localized or fully polarized electron bands depending on the magnetic state at a subset of orbitals coexisting with itinerant (bad-metallic or not) electrons at the less correlated orbitals. This tendency is clearly visible in Fig. 6 where the majority $t_{2 g}$ bands are fully spin-polarized, while the $e_{g}-\uparrow$ channel remains close to half-filling. Interesting as well is the electronic reconstruction within the minority $t_{2 g^{2}}$ sector, which shows Mott localization at low-energies and Hubbard like features similar to that found in the paramagnetic state. Our results thus suggest the coexistence of distinct electronic and spin degrees of freedom in ferromagnetic $\mathrm{BiFeO}_{3}$. This behavior is expected for ferromagnetic systems close to electronic delocalization, where strong orbital and spin differentiation prevents the insulating fixed point. Our work calls for future photoemission and polarized optical studies on ferromagnetic

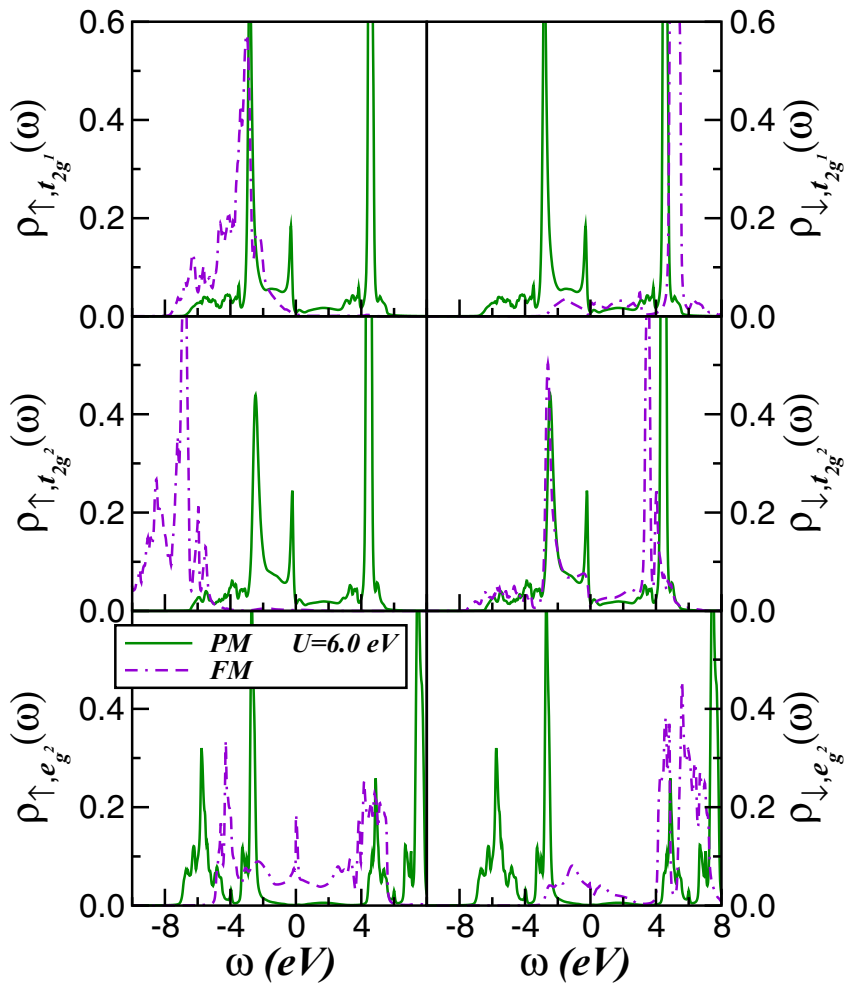

FIG. 6. Effect of ferromagnetic spin-ordering on the orbitaland spin-resolved electronic structure of $\mathrm{BiFeO}_{3}$ parent compound. Notice the large transfer of spectral weight yielding orbitaldifferentiated electronic localization and metallicity within the $t_{2 g}$ and $e_{g}$ orbital sectors.

$\mathrm{BiFeO}_{3}$. These studies will constitute a proof to anisotropic electronic delocalization and nonglobal electron-frozen ferromagnetism as well as the importance of treating dynamical correlations adequately to reveal a variety of unexplored pathways of designing metallic ferromagnets for future spintronic devices [60].

Taken together, this work have shown how one- and twoparticle features can semiquantitatively be understood within our specific L(S)DA+DMFT(MO-IPT) proposal. The central message of our approach is thus that a strong coupling picture of $\mathrm{BiFeO}_{3}$ leads to a satisfying description of the evolution of the MO electronic structure over the whole range of interest for orbital reconstruction [29] of paramagnetic and ferromagnetic $\mathrm{BiFeO}_{3}$. Clearly, being based on a first-principles correlated approach involving the full spin-resolved $\mathrm{MO}$ electronic structure of ferromagnetic $\mathrm{BiFeO}_{3}$, our proposal goes way beyond earlier antiferro phase work [14], where only the total valence band spectra have been compared, showing negligible electronic reconstruction between para- to antiferromagnetic phases. Differently to that recent LDA+DMFT(CT-QMC) study, our results show strong electronic orbital reconstruction between the magnetic phases. Importantly, in the ferromagnetic regime, an itinerant-localized duality underpins the physical behavior of the system, simultaneously giving rise to metallic bands and to dynamically fluctuating local moments putting ferromagnetic $\mathrm{BiFeO}_{3}$ close to selective Mottness. Based on our results in Fig. 6, we predict coexistence of 
residual pseudogaped states and narrow Kondo quasiparticles in metallic $\mathrm{BiFeO}_{3}$. Characteristic strong correlation features like self-energy corrections, local moment formation, and Kondo clouds are all predicted to occur towards metallizing the Mott insulator. Observation of these features would constitute further proof of the strongly correlated $\mathrm{MO}$ nature of $\mathrm{BiFeO}_{3}$ bulk and films relevant for magnetoelectronics [30].

\section{CONCLUSION}

In this paper, we have performed LDA+DMFT calculations for the multi-orbital Hubbard model to provide a microscopic description of the excitation spectrum which emerges within the paramagnetic and ferromagnetic ordered state of rhombohedral $\mathrm{BiFeO}_{3}$. Strong electron localization with pronounced Mott gap and Hubbard bands is found within the paramagnetic insulating state using realistic values for the on-site Coulomb interaction. Additionally, we show how multiorbital electron-electron interactions induce an orbitalselective electronic state [48] for ferromagnetic $\mathrm{BiFeO}_{3}$ with coexisting ordered magnetic moments and delocalized electronic states. The good qualitative accord between our numerical results with experimental hard $\mathrm{x}$-ray photoelectron spectroscopy (HAXPES) of bulk [14] and $\mathrm{BiFeO}_{3}$ thick films [22] as well as with $\mathrm{O} K$-edge $\mathrm{x}$-ray absorption (XAS) spectra [24,54] provides support to our proposal of orbital-selective metallization and spin ordering in ferromagnetic $\mathrm{BiFeO}_{3}$. The central message of our approach is thus to show that a strong coupling picture leads to a satisfying description for the insulating gap and the electronic structure reconstruction over the range of interest for optical and band-gap experiments. Clearly, being based on a first-principles correlated LDA+DMFT approach involving the valence and conduc- tion band electronic structure of $\mathrm{BiFeO}_{3}$, our proposal goes beyond earlier theoretical work [14], which focused solely on HAXPES and the correlated valence band spectra. In the strong coupling regime, itinerant-localized duality underpins the physical behavior of ferromagnetic $\mathrm{BiFeO}_{3}$, simultaneously giving rise to dynamically fluctuating local moments coexisting with itinerant electrons at less correlated orbitals, putting the ferromagnetic phase close to spin-selective Mottness [61]. Taken together, our study provides further support for the strongly correlated nature of $\mathrm{BiFeO}_{3}$ and it is expected to be generally applicable to understanding orbital-selective spin ordering [62] in strongly correlated electron systems and the underlying electronic state, which might emerge in ferroelectric, magnetically ordered spintronic devices [63].

\section{ACKNOWLEDGMENTS}

L.C.'s work is supported by CNPq (Grant No. 304035/2017-3). L.C. and S.S.C. wish to thank R. J. Prado for discussions regarding $\mathrm{BiFeO}_{3}$ films before this study was conceived. S.L. acknowledges support from the UK Research Council for using work in the paper that was undertaken by a student under Project No. EP/M50631X/1 as well as the DFG for support under the priority project SPP1415 and for a personal Heisenberg grant. S.L. also thanks ARCCA Cardiff for computational resources. Via S.L.'s membership of the UK's HPC Materials Chemistry Consortium, which is funded by EPSRC (No. EP/L000202), this work made use of the facilities of ARCHER, the UK's National High-Performance Computing Service, which is funded by the Office of Science and Technology through EPSRC's High End Computing Programme.
[1] See, for example, N. A. Spaldin and M. Fiebig, Science 309, 391 (2005); M. Feibig, T. Lottermoser, D. Meier, and M. Trassin, Nat. Rev. Mater. 1, 16046 (2016); K. Shimamoto, S. Mukherjee, S. Manz, J. S. White, M. Trassin, M. Kenzelmann, L. Chapon, T. Lippert, M. Fiebig, C. W. Schneider, and C. Niedermayer, Sci. Rep. 7, 44753 (2017); and references therein.

[2] J. T. Heron, J. L. Bosse, Q. He, Y. Gao, M. Trassin, L. Ye, J. D. Clarkson, C. Wang, Jian Liu, S. Salahuddin, D. C. Ralph, D. G. Schlom, J. Iñiguez, B. D. Huey, and R. Ramesh, Nature (London) 516, 370 (2014).

[3] J. Wang, J. B. Neaton, H. Zheng, V. Nagarajan, S. B. Ogale, B. Liu, D. Viehland, V. Vaithyanathan, D. G. Schlom, U. V. Waghmare, N. A. Spaldin, K. M. Rabe, M. Wuttig, and R. Ramesh, Science 299, 1719 (2003).

[4] L. Bi, A. R. Taussig, H.-S. Kim, L. Wang, G. F. Dionne, D. Bono, K. Persson, G. Ceder, and C. A. Ross, Phys. Rev. B 78, 104106 (2008).

[5] A. Feroze, M. Idrees, D.-K. Kim, M. Nadeem, S. A. Siddiqi, S. F. Shaukat, M. Atif, and M. Siddique, J. Electron. Mater. 46, 4582 (2017).

[6] R. Seshadri and N. A. Hill, Chem. Mater. 13, 2892 (2001).
[7] C. Ederer and N. A. Spaldin, Phys. Rev. B 71, 060401(R) (2005).

[8] J. B. Neaton, C. Ederer, U. V. Waghmare, N. A. Spaldin, and K. M. Rabe, Phys. Rev. B 71, 014113 (2005).

[9] S. L. Shang, G. Sheng, Y. Wang, L. Q. Chen, and Z. K. Liu, Phys. Rev. B 80, 052102 (2009).

[10] S. Ju, T.-Y. Cai, and G.-Y. Gou, J. Chem. Phys. 130, 214708 (2009).

[11] D. Albrecht, S. Lisenkov, W. Ren, D. Rahmedov, I. A. Kornev, and L. Bellaiche, Phys. Rev. B 81, 140401(R) (2010).

[12] M. K. Yaakob, M. F. M. Taib, L. Lu, O. H. Hassan, and M. Z. A. Yahya, Mater. Res. Express 2, 116101 (2015).

[13] A. O. Shorikov, A. V. Lukoyanov, V. I. Anisimov, and S. Y. Savrasov, Phys. Rev. B 92, 035125 (2015).

[14] S. Paul, D. Iusan, P. Thunström, Y. O. Kvashnin, J. Hellsvik, M. Pereiro, A. Delin, R. Knut, D. Phuyal, A. Lindblad, O. Karis, B. Sanyal, and O. Eriksson, Phys. Rev. B 97, 125120 (2018).

[15] S. V. Kiselev, R. P. Ozerov, and G. S. Zhdanov, Sov. Phys. Dokl. 7, 472 (1963).

[16] J. R. Teague, R. Gerson, and W. James, Solid State Commun. 8, 1073 (1970).

[17] F. Kubel and H. Schimid, Acta Crystallogr. Sect. B 46, 698 (1990). 
[18] A. Palewicz et al., Acta Phys. Pol. A 117, 296 (2009).

[19] J. Liu, M. Li, L. Pei, J. Wang, Z. Hu, X. Wang, and X. Zhao, Europhys. Lett. 89, 57004 (2010).

[20] J. Wu, S. Mao, Z.-G. Ye, Z. Xiea, and L. Zhenga, J. Mater. Chem. 20, 6512 (2010).

[21] S. Gómez-Salces, F. Aguado, F. Rodrí guez, R. Valiente, J. González, R. Haumont, and J. Kreisel, Phys. Rev. B 85, 144109 (2012).

[22] D. Mazumdar, R. Knut, F. Thöle, M. Gorgoi, S. Faleev, O. N. Mryasov, V. Shelke, C. Ederer, N. A. Spaldin, A. Gupta, and O. Karis, J. Electron Spectrosc. Relat. Phenom. 208, 63 (2016).

[23] M. M. Shirolkar, X. Dong, J. Li, S. Yin, M. Lia, and H. Wang, Phys. Chem. Chem. Phys. 18, 25409 (2016).

[24] Y. Ting, C.-S. Tu, P.-Y. Chen, C.-S. Chen, J. Anthoniappen, V. H. Schmidt, J.-M. Lee, T.-S. Chan, W.-Y. Chen, and R.-W. Song, J. Mater. Sci. 52, 581 (2017).

[25] B. Ruette, S. Zvyagin, A. P. Pyatakov, A. Bush, J. F. Li, V. I. Belotelov, A. K. Zvezdin, and D. Viehland, Phys. Rev. B 69, 064114 (2004).

[26] M. Bibes, Nat. Mater. 11, 354 (2012).

[27] C. Michel, J.-M. Moreau, G. D. Achenbach, R. Gerson, and W. J. James, Solid State Commun. 7, 701 (1969).

[28] See also, J. Zhang, M. A. Gondal, W. Wei, T. Zhang, Q. $\mathrm{Xu}$, and K. Shen, J. Alloys Compd. 530, 107 (2012); N. An, H.-T. Zhang, C.-Z. Liu, C.-B. Fan, X. Dong, Q.-L. Song, and G. Y. Wen, Key Eng. Mater. 727, 996 (2017); D. Bilican, E. Menendez, J. Zhang, P. Solsona, J. Fornell, E. Pellicer, and J. Sort, RSC Adv. 7, 32133 (2017).

[29] P. Yu, J.-S. Lee, S. Okamoto, M. D. Rossell, M. Huijben, C.-H. Yang, Q. He, J. X. Zhang, S. Y. Yang, M. J. Lee, Q. M. Ramasse, R. Erni, Y.-H. Chu, D. A. Arena, C.-C. Kao, L. W. Martin, and R. Ramesh, Phys. Rev. Lett. 105, 027201 (2010).

[30] R. Ramesh and N. A. Spaldin, Nat. Mater. 6, 21 (2007); see also, G. A. Prinz, Science 282, 1660 (1998).

[31] V. Fruth, E. Tenea, M. Gartner, M. Anastasescu, D. Berger, R. Ramer, and M. Zaharescu, J. Eur. Ceram. Soc. 27, 937 (2007).

[32] T. P. Gujar, V. R. Shinde, and C. D. Lokhande, Mater. Chem. Phys. 103, 142 (2007).

[33] G. Kotliar, S. Y. Savrasov, K. Haule, V. S. Oudovenko, O. Parcollet, and C. A. Marianetti, Rev. Mod. Phys. 78, 865 (2006).

[34] O. K. Andersen, Phys. Rev. B 12, 3060 (1975).

[35] For the implementation of the LMTO scheme see V. Antonov, B. Harmon, and A. Yaresko, Electronic Structure and MagnetoOptical Properties of Solids (Kluwer, Dordrecht, Boston, London, 2004).

[36] S. Chadov, X. Qi, J. Kübler, G. H. Fecher, C. Felser, and S. C. Zhang, Nat. Mater. 9, 541 (2010); L. Craco and S. Leoni, Sci. Rep. 5, 13772 (2015); S. V. Borisenko, D. V. Evtushinsky, Z.-H. Liu, I. Morozov, R. Kappenberger, S. Wurmehl, B. Büchner, A. N. Yaresko, T. K. Kim, M. Hoesch, T. Wolf, and N. D. Zhigadlo, Nat. Phys. 12, 311 (2016).

[37] L. Craco, M. S. Laad, S. Leoni, and E. Müller-Hartmann, Phys. Rev. B 70, 195116 (2004).

[38] L. Craco, S. Leoni, M. S. Laad, and H. Rosner, Phys. Rev. B 76, 115128 (2007).

[39] See, for example, L. Craco, M. S. Laad, and E. MüllerHartmann, Phys. Rev. B 74, 064425 (2006); M. S. Laad and L. Craco, Phys. Rev. Lett. 103, 017002 (2009); L. Craco and S. Leoni, Sci. Rep. 7, 46439 (2017).
[40] L. Qiang, H. Duo-Hui, C. Qi-Long, and W. Fan-Hou, Chin. Phys. B 22, 037101 (2013).

[41] The subscript one and two denote the orbital degeneracy of the $t_{2 g}$ and $e_{g}$ orbitals intrinsic to perovskite $\mathrm{BiFeO}_{3}$. For the $R 3 c$ phase orbitals can also be considered as twofold degenerate $e_{g}^{\pi}, e_{g}^{\sigma}$ subbands and a nondegenerate $a_{1 g}$ band [13].

[42] M. Imada, A. Fujimori, and Y. Tokura, Rev. Mod. Phys. 70, 1039 (1998).

[43] L. Craco, Phys. Rev. B 77, 125122 (2008).

[44] H. Kajueter and G. Kotliar, Phys. Rev. Lett. 77, 131 (1996).

[45] A. Georges, G. Kotliar, W. Krauth, and M. J. Rozenberg, Rev. Mod. Phys. 68, 13 (1996); see also, M. S. Laad, L. Craco, and E. Müller-Hartmann, Phys. Rev. B 67, 033105 (2003).

[46] P. Pou, R. Pérez, F. Flores, A. L. Yeyati, A. Martin-Rodero, J. M. Blanco, F. J. García-Vidal, and J. Ortega, Phys. Rev. B 62, 4309 (2000).

[47] N. Dasari, W. R. Mondal, P. Zhang, J. Moreno, M. Jarrell, and N. S. Vidhyadhiraja, Eur. Phys. J. B 89, 202 (2016).

[48] R. Yu and Q. Si, Phys. Rev. Lett. 110, 146402 (2013).

[49] M. S. Laad, L. Craco, S. Leoni, and H. Rosner, Phys. Rev. B. 79, 024515 (2009); see also, L. Craco and J. L. B. Faria, J. Appl. Phys. 119, 085107 (2016).

[50] See, A. Gössling, R. Schmitz, H. Roth, M. W. Haverkort, T. Lorenz, J. A. Mydosh, E. Müller-Hartmann, and M. Grüninger, Phys. Rev. B 78, 075122 (2008); and references therein.

[51] N. E. Massa, L. del Campo, D. De S. Meneses, P. Echegut, G. F. L. Fabbris, G. de M. Azevedo, M. J. Martínez-Lope, and J. A. Alonso, J. Appl. Phys. 108, 084114 (2010).

[52] L. de' Medici, G. Giovannetti, and M. Capone, Phys. Rev. Lett. 112, 177001 (2014).

[53] P. S. V. Mocherla, C. Karthik, R. Ubic, M. S. R. Rao, and C. Sudakar, Appl. Phys. Lett. 103, 022910 (2013).

[54] R. Sæterli, S. M. Selbach, P. Ravindran, T. Grande, and R. Holmestad, Phys. Rev. B 82, 064102 (2010).

[55] L. Craco and E. Müller-Hartmann, Phys. Rev. B 77, 045130 (2008); L. Craco, M. S. Laad, and S. Leoni, J. Phys.: Conf. Ser. 487, 012017 (2014); M. D. Watson, S. Backes, A. A. Haghighirad, M. Hoesch, T. K. Kim, A. I. Coldea, and R. Valenti, Phys. Rev. B 95, 081106(R) (2017).

[56] P. S. Cornaglia and A. Georges, Phys. Rev. B 75, 115112 (2007).

[57] B. Freelon, Y. H. Liu, J.-L. Chen, L. Craco, M. S. Laad, S. Leoni, J. Chen, L. Tao, H. Wang, R. Flauca, Z. Yamani, M. Fang, C. Chang, J.-H. Guo, and Z. Hussain, Phys. Rev. B 92, 155139 (2015)

[58] L. Craco, Phys. Rev. B 96, 165412 (2017).

[59] I. Muneta, S. Ohya, H. Terada, and M. Tanakac, Nat. Commun. 7, 12013 (2016).

[60] H. Ohno, Nat. Mater. 9, 952 (2010).

[61] J. Faúndez, T. N. Jorge, and L. Craco, Phys. Rev. B 97, 115149 (2018).

[62] J. M. Caron, J. R. Neilson, D. C. Miller, K. Arpino, A. Llobet, and T. M. McQueen, Phys. Rev. B 85, 180405(R) (2012); L. Craco and S. S. Carara, ibid. 97, 205114 (2018).

[63] R. Ramesh, Nat. Mater. 9, 380 (2010); see also, Y. F. Wu, H. D. Song, L. Zhang, X. Yang, Z. Ren, D. Liu, H. C. Wu, J. Wu, J. G. Li, Z. Jia, B. Yan, X. Wu, C. G. Duan, G. Han, Z. M. Liao, and D. Yu, Phys. Rev. B 95, 195426 (2017). 